\title{
Limitations of deterministic auction design for correlated bidders *
}

\author{
Ioannis Caragiannis ${ }^{\dagger} \quad$ Christos Kaklamanis $^{\dagger} \quad$ Maria Kyropoulou $^{\ddagger}$
}

March 19, 2016

\begin{abstract}
The seminal work of Myerson (Mathematics of OR '81) characterizes incentive-compatible single-item auctions among bidders with independent valuations. In this setting, relatively simple deterministic auction mechanisms achieve revenue optimality. When bidders have correlated valuations, designing the revenue-optimal deterministic auction is a computationally demanding problem; indeed, Papadimitriou and Pierrakos (STOC '11) proved that it is APX-hard, obtaining an explicit inapproximability factor of $1999 / 2000=$ $99.95 \%$. In the current paper, we strengthen this inapproximability factor to $63 / 64 \approx$ 98.5\%. Our proof is based on a gap-preserving reduction from the MAX-NM 3SAT problem; a variant of the maximum satisfiability problem where each clause has exactly 3 literals and no clause contains both negated and unnegated literals. We furthermore show that the gap between the revenue of deterministic and randomized auctions can be as low as $13 / 14 \approx 92.9 \%$, improving an explicit gap of $947 / 948 \approx 99.9 \%$ by Dobzinski, $\mathrm{Fu}$, and Kleinberg (STOC '11).
\end{abstract}

\section{Introduction}

In the classical model of Auction Theory [Krishna, 2009], a seller auctions off an item to $n$ bidders with valuations for the item drawn independently from known but not necessarily identical probability distributions. Myerson's seminal work 1981 gives an elegant characterization of revenue-maximizing auctions in this setting. Optimal revenue is achieved by simple deterministic auctions that are defined using succinct information about the probability distributions. In contrast, the case of bidders with correlated valuations has been a mystery; in spite of the vast related literature in Economics and Computer Science, no such general characterization result has been presented so far. Ronen [2001] was the first to consider an inspirational alternative approach and provided an auction that approximates the revenue of the optimal auction, contrary to the prevailing literature that was struggling for a characterization. More recently, Papadimitriou and Pierrakos [2011] provided an explanation — from the computational complexity point of view - for the necessity of this alternative approach

${ }^{*}$ A preliminary version of this paper appeared in Proceedings of the 21st Annual European Symposium on Algorithms (ESA), LNCS 8125, Springer, pages 277-288, 2013. This work was partially supported by the European Social Fund and Greek national funds through the research funding program Thales on "Algorithmic Game Theory", by "Caratheodory" research grant E.114 from the University of Patras, and by ERC Advanced Grant 321171 (ALGAME).

†University of Patras and CTI "Diophantus", Greece

${ }^{\ddagger}$ University of Oxford, UK 
for the case of deterministic auctions by proving that the problem of designing the optimal deterministic auction given the explicit description of the joint probability distribution (with finite support) is APX-hard. Due to their simplicity and amenability to implement in practice, deterministic auctions are of particular importance. Furthermore, Dobzinski et al. [2011] provided a separation between randomized (truthful in expectation) and deterministic truthful auctions: there are (single-item) settings in which randomized auctions may extract strictly more revenue than any deterministic auction. Both results hold even when only three bidders participate in the auction. In this paper, we strengthen both results.

Existing approaches to single-item auctions with non-independent bidders fall into three different categories. A first approach that has been mostly followed by economists (e.g, see [Constantin et al., 2007; Levin and Smith, 1996; Maskin, 2003; Milgrom and Weber, 1982]) assumes that each bidder has her own valuation function that depends on a shared random variable; this model is usually referred to as the interdependent valuations model. In a second approach, the support of the joint probability distribution is extremely large (exponentially larger than the number of players or even infinite) and an auction mechanism can obtain information about the distribution through queries (e.g, see [Dobzinski et al., 2011; Ronen, 2001; Ronen and Saberi, 2002]). The related literature focuses on the design of auctions that use only a polynomial (in terms of the number of bidders) number of queries. In the third one, the joint probability has finite support and the related work seeks for auctions that are defined in polynomial time in terms of the support size and the number of players (e.g, see [Dobzinski et al., 2011; Papadimitriou and Pierrakos, 2011]). The last two models are known as the query model and the explicit model, respectively. Among these models, the explicit one allows us to view the design of the revenue-optimal (deterministic) auction as a standard optimization problem. The auction has to define the bidder that gets the item and her payment to the auctioneer for every valuation vector of the support of the joint probability distribution. Both the allocation and the payments should be defined in such a way that no bidder has an incentive to misreport her true valuation; this constraint is known as incentive compatibility. The objective is to maximize the expected revenue of the auctioneer over all valuation vectors.

Since our purpose is to explore the limitations of deterministic auctions, we focus on the explicit model and the case of three bidders. Following [Papadimitriou and Pierrakos, 2011], we refer to the optimization problem mentioned above (when restricted to three bidders) as 3OptimalAuctionDesign. The inapproximability bound presented by Papadimitriou and Pierrakos [2011] is marginally smaller than 1 , namely $1999 / 2000>99.9 \%$. It is achieved by a gap-preserving reduction from a structured maximum satisfiability problem called CATSAT. This problem has an inapproximability of 79/80; hence, the gap obtained for 3OpTIMALAUCTIONDESIGN is even closer to 1 . We present a different reduction from MAX-NM 3SAT; a variant of the maximum satisfiability problem where each clause has exactly 3 literals and no clause contains both negated and unnegated literals. This problem is shown in [Guruswami and Khot, 2005] to maintain the 7/8 inapproximability factor of the classical MAX 3SAT problem. Our reduction yields a significantly improved inapproximability bound of $63 / 64 \approx 98.5 \%$ for 3 OptimalAuctionDesign ${ }^{1}$. Furthermore, we demonstrate a rather significant revenue

\footnotetext{
${ }^{1}$ We note that a significantly more involved reduction from the problem of maximizing the number of satisfied linear equations in an over-determined system of linear equations modulo 2 yields a slightly stronger inapproximability factor of $57 / 58 \approx 98.3$ as is shown in the conference version of the current paper [Caragiannis et al., 2013]. Following the suggestion of an anonymous reviewer, we have chosen to present the simpler reduction from MAX-NM 3SAT here.
} 
gap between deterministic truthful mechanisms and randomized auctions that are truthful in expectation; the revenue of any deterministic auction can be at most $13 / 14 \approx 92.9 \%$ of the optimal randomized one. This result improves the previously known explicit bound of $947 / 948 \approx 99.9 \%$ of Dobzinski et al. [2011]. Our construction is considerably simpler than the one in [Dobzinski et al., 2011].

Related work Extending Myerson's work, Crémer and McLean [1985; 1988] characterize the information structure that guarantees the auctioneer full surplus, under several settings with correlated valuations. They consider interim individual rationality which allows players to have negative utility for some valuation vectors. In contrast, our work (as well as more recent related work) focuses on ex post individual rationality, a design requirement that does not allow such situations. Ronen [2001] and Ronen and Saberi [2002] consider single-item optimal auctions in the query model. They design auctions that use queries of the following form: given the valuations of a set of players, what is the conditional distribution of the remaining ones? The 1-lookahead auction in [Ronen, 2001] yields at least half the optimal revenue to the seller. Essentially, the auction ignores the $n-1$ lowest bids and offers the item to the remaining bidder at the price that maximizes the revenue considering the distribution of valuations of that bidder conditioned on the valuations of everybody else. Ronen and Saberi [2002] present several impossibility results for auctions of particular type. For example, they prove that no ascending auction can approximate the optimal revenue to a factor greater than $7 / 8$.

Dobzinski et al. [2011] consider $k$-lookahead auctions (a natural extension of 1-lookahead) and show that a $2 / 3$-approximation of the optimal revenue can be achieved by randomized auctions in the query model. For the explicit model, they show that the optimal randomized auction can be computed by linear programming while the deterministic 2-lookahead auction achieves a 3/5-approximation of revenue. Their positive results have been strengthened by Chen et al. [2011] to approximation factors of 0.731 and 0.622, respectively. Both Dobzinski et al. [2011] and Papadimitriou and Pierrakos [2011] prove that revenue-optimal auction design can be solved in polynomial time in the 2-bidder case. The 2-bidder case has also been considered in [Esö, 2005] and [Diakonikolas et al., 2012]. In particular, Diakonikolas et al. [2012] study the tradeoff between efficiency and revenue in deterministic truthful auctions and prove that any point of the Pareto curve can be approximated with arbitrary precision. Roughgarden and Talgam-Cohen [2013] examine the assumptions that are required for an analog to the Myerson's optimal auction theory to apply to several interdependent valuation models. In particular for the correlated private valuations case they show that regularity and affiliation of the distributions of valuations (infinite support) are sufficient conditions.

Roadmap The rest of the paper is structured as follows. We begin with preliminary definitions in Section 2. The reduction and the proof of the inapproximability of 3OptimalAucTIONDESIGN are presented in Sections 3 and 4, respectively. The revenue-gap construction is presented in Section 5.

\section{Preliminaries}

We consider having a single indivisible item for sale and $n$ players competing for that item. Each player has a finite number of possible (scalar) valuations for the item, but the valuations 
of all players are correlated. Let $\mathcal{D}$ denote the joint probability distribution over valuation vectors $\mathbf{v}=\left(v_{1}, \ldots, v_{n}\right)$, where $v_{i}$ denotes the valuation of player $i$. An auction mechanism $\mathcal{M}$ decides the probability $x_{i}(\mathbf{v})$ that player $i$ is allocated the item, and her payment $p_{i}(\mathbf{v})$ in advance, for every valuation vector in the support of $\mathcal{D}$. The allocation probabilities can be considered as allocating fractions of the item to players under the restriction that the total allocation fraction is at most 1 for every possible valuation vector $\mathbf{v}$, i.e., $\sum_{i} x_{i}(\mathbf{v}) \leq 1$.

The mechanism has no way of knowing which valuation vector is eventually realized, and the actual outcome is based on the declarations of valuations of the players (bids). Whenever the players bid as in $\mathbf{b}=\left(b_{1}, \ldots, b_{n}\right)$, the (expected) utility of player $i$ is defined as

$$
u_{i}(\mathbf{b})=x_{i}(\mathbf{b}) \cdot v_{i}-p_{i}(\mathbf{b}) .
$$

Players are assumed to be utility-maximizers which implies that a player declares the valuation that maximizes her utility given the bids of everybody else. We adopt the point of view of the auctioneer and examine the problem of designing an auction mechanism that satisfies some desirable properties. Incentive compatibility, individual rationality, and the no-positivetransfers property are classically considered as important desiderata for auction mechanisms [Krishna, 2009; Nisan et al., 2007], and revenue maximization is an obvious objective. In what follows we introduce some additional notation so that we can formally define these notions.

We use $\mathbf{v}_{-i}$ to denote the vector of valuations of all players but $i$, and $\left(v_{i}^{\prime}, \mathbf{v}_{-i}\right)$ to denote the valuation vector that is derived by $\mathbf{v}$ if only player $i$ 's valuation is swapped from $v_{i}$ to $v_{i}^{\prime}$; note that $\mathbf{v}=\left(v_{i}, \mathbf{v}_{-i}\right)$. The incentive compatibility property requires that truth-telling maximizes players' utility, i.e., for every player $i, v_{i}, \mathbf{v}_{-i}$ and $v_{i}^{\prime}$, it holds that $u_{i}(\mathbf{v}) \geq u_{i}\left(v_{i}^{\prime}, \mathbf{v}_{-i}\right)$ and, equivalently,

$$
x_{i}(\mathbf{v}) \cdot v_{i}-p_{i}(\mathbf{v}) \geq x_{i}\left(v_{i}^{\prime}, \mathbf{v}_{-i}\right) \cdot v_{i}-p_{i}\left(v_{i}^{\prime}, \mathbf{v}_{-i}\right) .
$$

Henceforth, we assume each player always submits as bid her actual valuation for the item, since vector $\mathbf{b}(\mathbf{v})=\mathbf{v}$ is utility-maximizing for all players given an incentive compatible mechanism; we also refer to such mechanisms as truthful mechanisms. The individual rationality property encourages players to participate in the auction. In particular, we consider ex post individual rationality that requires that players always have non-negative utility. In other words, for every player $i$ and valuation vector $\mathbf{v}$, it holds that

$$
x_{i}(\mathbf{v}) \cdot v_{i}-p_{i}(\mathbf{v}) \geq 0 .
$$

Finally, the no-positive-transfers property implies that the players never receive payments from the auctioneer, i.e.,

$$
p_{i}(\mathbf{v}) \geq 0
$$

for every player $i$ and valuation vector $\mathbf{v}$.

As we have mentioned above, an obvious objective for auction mechanisms is the maximization of the expected revenue, i.e., the expectation over all valuation vectors of the payment received by the auctioneer. Let $\mathbf{p}$ denote the payments applied by mechanism $\mathcal{M}$. The revenue derived by $\mathcal{M}$ is computed as follows:

$$
\operatorname{rev}(\mathcal{M})=\mathbb{E}_{\mathbf{v} \sim \mathcal{D}}\left[\sum_{i} p_{i}(\mathbf{v})\right] .
$$

We note that the revenue-maximizing truthful-in-expectation mechanism in the explicit setting of correlated valuations can be computed in polynomial time by a linear program [Dobzinski et al., 2011]. 
In the more restricted setting where the possible valuations of player $i$, for $i=1, \ldots, n$, must form a continuous interval, it is a well-known fact (see Chapter 13 of [Nisan et al., 2007]) that a mechanism is truthful (in expectation) if and only if the following two conditions hold: the allocation $x$ is monotone (i.e., for every player $i, v_{i}, \mathbf{v}_{-i}$, and $v_{i}^{\prime} \geq v_{i}$ it holds that $\left.x_{i}\left(v_{i}^{\prime}, \mathbf{v}_{-i}\right) \geq x_{i}(\mathbf{v}),\right)$ and the payments are computed according to $p_{i}(\mathbf{v})=$ $x(\mathbf{v}) \cdot v_{i}-\int_{0}^{v_{i}} x_{i}\left(z, \mathbf{v}_{-i}\right) \mathrm{d} z$. In the explicit setting of correlated valuations that we examine, the distribution of valuations has finite support. In this case, we can limit our search to monotone allocations and a specific payment function, using the following observation.

Observation 1 A randomized, single-item auction mechanism $\mathcal{M}$ satisfies the following:

- If $\mathcal{M}=(x, \mathbf{p})$ is truthful in expectation, then

- allocation $x$ is monotone, i.e., for all $i, \mathbf{v}_{-i}, v_{i}$, and $v_{i}^{\prime}>v_{i}$, it holds that

$$
x_{i}\left(v_{i}^{\prime}, \mathbf{v}_{-i}\right) \geq x_{i}(\mathbf{v})
$$

- for a given monotone allocation $x$, the maximum revenue is achieved when assigning payments

$$
p_{i}\left(v_{i}^{\lambda}, \mathbf{v}_{-i}\right)=x_{i}\left(v_{i}^{\lambda}, \mathbf{v}_{-i}\right) \cdot v_{i}^{\lambda}-\sum_{k=1}^{\lambda-1} x_{i}\left(v_{i}^{k}, \mathbf{v}_{-i}\right) \cdot\left(v_{i}^{k+1}-v_{i}^{k}\right),
$$

where $v_{i}^{\lambda}$ is the $\lambda$-th possible valuation of $i$ such that $\left(v_{i}^{\lambda}, \mathbf{v}_{-i}\right)$ occurs with positive probability

- If $\mathcal{M}=(x, \mathbf{p})$ satisfies that allocation $x$ is monotone and $\mathbf{p}$ is defined as above, then $\mathcal{M}$ is truthful in expectation

For completeness, we provide the proof of this observation in Appendix A. Notice that a monotone allocation implies payments that satisfy $p_{i}\left(v_{i}^{\lambda}, \mathbf{v}_{-i}\right) \geq x\left(v_{i}^{\lambda}, \mathbf{v}_{-i}\right) \cdot v_{i}^{s}$, where $v_{i}^{s}$ is the smallest valuation such that $x\left(v_{i}^{s}, \mathbf{v}_{-i}\right)>0$, i.e., player $i$ has non-zero probability of getting the item. Also, note that, given a monotone allocation, applying payments according to equality (1) implies individual rationality and the no-positive-transfers property. As we are only interested in truthful mechanisms that satisfy these properties and maximize revenue, we can assume that a mechanism $\mathcal{M}$ is entirely defined by its allocation $A$ and assigns payments according to equality (1); we denote the revenue of $\mathcal{M}$ as rev $(A)$. Moreover, it suffices to consider only monotone allocations.

In Sections 3 and 4, we focus on deterministic auction mechanisms, where the allocation probabilities satisfy $x_{i}(\mathbf{v}) \in\{0,1\}$, for $i=1, \ldots, n$. The search space is now restricted to (deterministic) monotone allocations and corresponding threshold payment functions; their definition can be made more intuitive in the deterministic setting (e.g., see [Papadimitriou and Pierrakos, 2011]) as follows. An allocation is monotone if when the item is allocated to some player $i$ for some valuation vector $\mathbf{v}$, i.e., $x_{i}(\mathbf{v})=1$, then player $i$ is also allocated the item for $\left(v_{i}^{\prime}, \mathbf{v}_{-i}\right)$ with $v_{i}^{\prime}>v_{i}$, i.e., $x_{i}\left(v_{i}^{\prime}, \mathbf{v}_{-i}\right)=1$ for any $v_{i}^{\prime}>v_{i}$. A threshold payment for a winning player $i$ is then defined as the infimum valuation $v_{i}^{\prime \prime}$ so that player $i$ gets the item for the valuation vector $\left(v_{i}^{\prime \prime}, \mathbf{v}_{-i}\right)$.

We consider auctions with three players and adopt a geometric representation of the problem that first appeared in [Papadimitriou and Pierrakos, 2011]. We assume that $\mathcal{D}$ is 
defined by a set $S$ of points in $\mathbb{R}^{3}$ and consider weights associated with these points. We refer to the three players as player $x, y$, and $z$; a point of $S$ corresponds to a valuation vector where players $x, y$, and $z$ have as valuation the $x-, y$-, and $z$-coordinate of the point. The weight of a point indicates the probability that the corresponding valuation vector is realized. Naturally, we refer to allocations of points to players to imply which player is allocated the item whenever the particular valuation vector (point) is realized. In the following, we say that two points are $x$-aligned (resp., $y$-aligned, $z$-aligned) if they have the same $y$ - and $z$-coordinates (resp., $x$ - and $z$-coordinates, $x$ - and $y$-coordinates). Given a deterministic mechanism, monotonicity implies that if a point $p$ is allocated to a player (say, $x$ ), then all points that are $x$-aligned with $p$ and have higher $x$-coordinate are enforced to be allocated to player $x$ as well (similarly for the other players). The payment associated with a point $p$ that is allocated to player $x$ is then the lowest $x$-coordinate of the $x$-aligned points with $p$ that are allocated to $x$ (similarly for the other players). For randomized allocations, where the allocation of points is fractional, the monotonicity requirement and the payments are correspondingly defined.

The problem of designing the optimal deterministic auction mechanism is stated as follows:

3OptimalAuctionDesign Given a finite set of points $S \subset \mathbb{R}^{3}$ and associated weights, compute a monotone allocation of the points of $S$ to players $x, y$, and $z$ so that the weighted sum of the implied threshold payments (expected revenue) is maximized.

\section{The inapproximability result - The construction}

In this section we present our gap-preserving reduction from MAX-NM 3SAT to 3OPTIMALAUCtionDESIGN.

MAX-NM 3SAT: Consider a set of $n$ binary variables $V_{1}, \ldots, V_{n}$ and a CNF formula $\Phi$ of $m$ clauses with exactly 3 literals per clause such that no clause is mixed, i.e., no clause contains both negated and unnegated literals. Find an assignment of the variables that maximizes the number of satisfied clauses.

Consider an instance of MAX-NM 3SAT, i.e., a CNF formula $\Phi$ of $m$ clauses on a set of $n$ variables $V_{i}$, for $i=1, \ldots, n$. Each clause of $\Phi$ is either of the form $\left(V_{h_{1}} \vee V_{h_{2}} \vee V_{h_{3}}\right)$ or of the form $\left(\neg V_{h_{1}} \vee \neg V_{h_{2}} \vee \neg V_{h_{3}}\right)$, with $1 \leq h_{1}<h_{2}<h_{3} \leq n$. We use the notation $c(h)$, for $h=1, \ldots, m$, to identify the clauses. Let $d_{i}$ be the degree of variable $V_{i}$, i.e., the number of equations in which $V_{i}$ participates either as a negated literal or as an unnegated one. We construct an instance $R(\Phi)$ of 3OptimalAuctionDesign such that the majority of the points lie inside a specified region whose boundaries are very close to 1 . Given the acceptable range of coordinates for the points of $R(I)$, it suffices to specify only their relative position to sketch the proof of our result. Providing such a description of the reduced construction serves two purposes; it keeps the presentation clear and at the same time gives a more intuitive overview of the construction. For completeness, right after the presentation of each gadget, we also give the exact coordinates of a set of points that formally prove our statements in specified paragraphs or tables.

Note that the constructed 3OptimalAUCTIONDESIGN instance has a polynomial number of points, and that both the coordinates of the points and their weights are rational numbers that require only polynomial precision. Without loss of generality, we consider weights that do not sum up to 1 and we consistently compute the revenue contributed by (the allocation of) a point as the product of the threshold payment and its weight. 
We are now ready to present our construction. $R(\Phi)$ contains one variable gadget and one clause gadget per clause. Most of the points of each of the gadgets have coordinates in $(1-\theta, 1+\theta)$ where $\theta \in(0,1 / 5600)$ is a very small constant. We refer to all these points as the main part of the construction. The only points that lie outside this region satisfy some particular properties and are called blockers. We begin with the description of blockers, which are extensively used afterwards.

\subsection{Blockers}

Our reduction uses a special class of points which we call blockers. The idea is that a blocker can prevent the allocation of a set of points to a certain player. Blockers essentially play the same role that scaffolding segments play in the reduction of [Papadimitriou and Pierrakos, 2011], but our reduction uses considerably fewer ones; this allows for a much smaller overhead in the optimal revenue and an improved inapproximability result.

Consider a set of $c \geq 1$ points $u_{i}$, for $i=1, \ldots, c$, that belong to the main part of the construction. Let $u_{i}, i=1, \ldots, c$, have weight $w_{i}$ and lie at $\left(x_{i}, y, z\right)$; the points are $x$-aligned, and all their coordinates $y, z$, and $x_{i}$, for $i=1, \ldots, c$, are at most $1+\theta$. Let $W$ denote their total weight, i.e, $W=\sum_{i=1}^{c} w_{i}$. Now, consider a point $b$ with negligibly small weight $\theta$ that is also $x$-aligned with the aforementioned points. Let $b$ be located exactly at $\left(x_{b}, y, z\right)$ where $x_{b}$ is such that $\theta x_{b}=(W+\theta)(1+\theta)$. The only way to obtain considerable revenue from $b$ is to allocate it to $x$; in any other case, the revenue derived by $b$ would be at most $\theta(1+\theta)$. Furthermore, allocating any other point among $u_{1}, u_{2}, \ldots, u_{c}$, together with $b$ to $x$ would not increase the revenue of the allocation. To see why this is true, observe that the contribution of $b$ to the revenue is $\theta x_{b}=(W+\theta)(1+\theta)$ if $b$ is allocated to $x$, whereas by allocating some of the other points and $b$ to player $x$, their contribution to the revenue is less than $(W+\theta)(1+\theta)$. We conclude that in a revenue-optimal allocation, $b$ is allocated to player $x$ and each point $u_{i}$ is allocated to either $y$ or $z$ or is not allocated at all; we say that $b x$-blocks the points $u_{1}, \ldots, u_{c}$, and we call $b$ a blocker. The properties of blockers that $y$-block or $z$-block other points are defined accordingly.

From this point on, since we are only interested in revenue-optimal allocations, we assume that blockers are always allocated towards their "preferred" direction and points that are blocked are never allocated towards their blocked direction (we ignore any other possibility whenever we consider the possible allocations of a specific set of points).

Our construction consists of several gadgets, i.e., one variable gadget and two kinds of clause gadgets. The variable gadget contains points that correspond to variables and have only two possible allocations (the third direction is blocked). These allocations correspond to setting the variable to 0 or 1 , respectively, in the original MAX-NM 3SAT instance. We also create one clause gadget for each clause of $\Phi$. There are several additional points (we call them connection points), which are insignificant in terms of the revenue they generate, but are very important in the following sense: the allocation of the connection points is completely determined by the allocation of the variable points and it affects the allocation of the points in the clause gadgets, hence the revenue that is generated by them. In other words, the allocation of the variable points completely determines the maximum attainable revenue from our construction, in a similar way as the assignment of values to variables determines the number of satisfiable clauses in the original instance. We now present these gadgets in more detail. 


\subsection{The variable gadget}

The role of the variable gadget is twofold. The allocation of some of the points indicates the assignment of values to the variables of the original instance $\Phi$, while other points aid the connection to the clause gadgets.

The variable gadget For every variable $V_{i}, i=1, \ldots, n$, the variable gadget has a variable point $v_{i}$ with weight $d_{i}(1 / 2+5 \theta)$. All these points are $x$-aligned with $(1,1,1)$ so that the $x$-coordinate of $v_{i+1}$ is higher than the $x$-coordinate of $v_{i}$. For each appearance of variable $V_{i}$ in a clause $c(h)$, there is one connection point $H_{i}(h)$ with weight $\theta$. Point $H_{i}(h)$ is either $y$-aligned of $z$-aligned with $v_{i}$ depending on whether $V_{i}$ appears as a negated or an unnegated literal in $c(h)$, respectively. The exact location of these points will become clear after the description of the clause gadgets. Recall that all the aforementioned points have coordinates in $[1,1+\theta)$. We also add a blocker $b$ that $x$-blocks the set of variable points.

Exact location Let $\epsilon=\theta / \max \{n+2, m+1\}$. The variable point $v_{i}$ is located at $(1+i \epsilon, 1,1)$ and has weight $d_{i}(1 / 2+5 \theta)$. The blocker $b$ is located at $((3 m(1 / 2+5 \theta)+\theta)(1+\theta) / \theta, 1,1)$ and has weight $\theta$. Regarding the $x$-coordinate of $b$, note that $\sum_{i=1}^{n} d_{i}=3 \mathrm{~m}$.

To give some intuition regarding the variable gadget, we note (and provide a formal proof later) that the weight $d_{i}(1 / 2+5 \theta)$ of point $v_{i}$ is significantly high so that at any revenueoptimal allocation, point $v_{i}$ should be allocated to either player $y$ or player $z$; these allocations correspond to setting variable $V_{i}$ to values 1 and 0 , respectively. Due to monotonicity, this enforces the allocation of the $y$-aligned or $z$-aligned connection points; intuitively, this propagates the fact that the variable $V_{i}$ is set to a certain value to the clause gadgets.

\subsection{The clause gadgets}

We continue by presenting the clause gadgets and clarify their connection to the variable gadget. For each clause of $\Phi$, we define a clause gadget of a particular type depending on whether the particular clause contains negated or unnegated literals. For two $x$-aligned points $p$ and $q$ of the main part of the construction, we use the notation $p_{(+x)} q$ and $p_{(-x)} q$ to denote that $q$ has larger and smaller $x$-coordinate than $p$, respectively (similarly for the other coordinates).

The clause gadget corresponding to clause $c(h)=\left(V_{i} \vee V_{j} \vee V_{k}\right)$ The clause gadget corresponding to $c(h)$ consists of the 5 -sequence of points $\left[A(h)_{(+x)} B(h)_{(-z)} C(h)_{(+x)}\right.$ $\left.D(h)_{(-z)} E(h)\right]$ and 2 blockers. All these points have $y$-coordinate in $(1-\theta, 1)$, i.e., they lie below the plane $y=1$. Points $A(h), C(h)$, and $E(h)$ have weight 1 , while points $B(h)$ and $D(h)$ have weight $\theta$. The points $A(h), C(h)$, and $E(h)$ are $y$-aligned to the connection points $H_{i}(h), H_{j}(h)$, and $H_{k}(h)$, respectively. Point $A(h)$ is $z$-blocked by blocker $b_{A(h)}$ while point $E(h)$ is $x$-blocked by blocker $b_{E(h)}$. See Figure 1 .

Exact location The exact location of the points comprising the clause gadget corresponding to clause $c(h)=\left(V_{i} \vee V_{j} \vee V_{k}\right)$ and the corresponding connection points towards the variable gadget appears at Table 1 .

We note that from this point on, we only consider blockers that are used to block a single point of weight 1 . So, we point out that they all have the same weight $\theta$ as well as the same 
Table 1: The points in the clause gadget associated with clause $c(h)=\left(V_{i} \vee V_{j} \vee V_{k}\right)$ and the corresponding connection points.

\begin{tabular}{|c||c|c|c||c|}
\hline Point & $c_{x}$ & $c_{y}$ & $c_{z}$ & Weight \\
\hline \hline$A(h)$ & $1+i \epsilon$ & $1-h \epsilon$ & $1+h \epsilon+2 \epsilon / 3$ & 1 \\
$B(h)$ & $1+j \epsilon$ & $1-h \epsilon$ & $1+h \epsilon+2 \epsilon / 3$ & $\theta$ \\
$C(h)$ & $1+j \epsilon$ & $1-h \epsilon$ & $1+h \epsilon+\epsilon / 3$ & 1 \\
$D(h)$ & $1+k \epsilon$ & $1-h \epsilon$ & $1+h \epsilon+\epsilon / 3$ & $\theta$ \\
$E(h)$ & $1+k \epsilon$ & $1-h \epsilon$ & $1+h \epsilon$ & 1 \\
\hline$b_{A}(h)$ & $1+i \epsilon$ & $1-h \epsilon$ & $(1+\theta)^{2} / \theta$ & $\theta$ \\
$b_{E}(h)$ & $(1+\theta)^{2} / \theta$ & $1-h \epsilon$ & $1+h \epsilon$ & $\theta$ \\
\hline$H_{i}(h)$ & $1+i \epsilon$ & 1 & $1+h \epsilon+2 \epsilon / 3$ & $\theta$ \\
$H_{j}(h)$ & $1+j \epsilon$ & 1 & $1+h \epsilon+\epsilon / 3$ & $\theta$ \\
$H_{k}(h)$ & $1+k \epsilon$ & 1 & $1+h \epsilon$ & $\theta$ \\
\hline
\end{tabular}

coordinate $(1+\theta)^{2} / \theta$ in the dimension that they block. Points with negligibly small weight $\theta$ that are part of the gadgets are crucial since they indirectly influence the allocation of unitweight points, e.g., $B(h)$ prevents $A(h)$ and $C(h)$ to be allocated to $x$ and $z$, respectively, at the same time. However, the allocation itself of these points does not contribute much to the revenue, since all possible threshold payments (all their coordinates) are at most $1+\theta$. In order to keep the analysis as simple as possible we focus on the allocation of unit-weight points for now, but note that the revenue of all points is accounted for at the end.

Consider a monotone allocation in which the connection points $H_{i}(h), H_{j}(h)$, and $H_{k}(h)$ are allocated to players $x$ or $z$. In this case, we say that the clause gadget is non-breathing in the sense that none of the points $A(h), C(h)$, and $E(h)$ can be allocated to player $y$. Hence, among the monotone allocations in which the clause gadget is non-breathing, the one that maximizes revenue leaves one of $A(h), C(h)$, and $E(h)$ unallocated. In contrast, if some of the connection points (say $H_{j}(h)$ ) is not allocated to players $x$ or $z$ (i.e., the clause gadget is breathing), the contribution of $A(h), C(h)$, and $E(h)$ can increase by allocating $A(h)$ and $B(h)$ to player $x, C(h)$ and $H_{j}(h)$ to player $y$, and $D(h)$ and $E(h)$ to player $z$. The other cases (i.e., when $H_{i}(h)$ or $H_{k}(h)$ are not allocated to the aforementioned players) have similar allocations of improved revenue.

The clause gadgets corresponding to clauses of the form $\left(\neg V_{i} \vee \neg V_{j} \vee \neg V_{k}\right)$ are symmetric and have identical properties. An exact description follows.

The clause gadget corresponding to clause $c(h)=\left(\neg V_{i} \vee \neg V_{j} \vee \neg V_{k}\right)$ The clause gadget corresponding to $c(h)$ consists of the 5-sequence of points $\left[A(h)_{(+x)} B(h)_{(-y)} C(h)_{(+x)}\right.$ $\left.D(h)_{(-y)} E(h)\right]$ and 2 blockers. All these points have $z$-coordinate in $(1-\theta, 1)$, i.e., they lie behind the plane $z=1$. Points $A(h), C(h)$, and $E(h)$ have weight 1 , while points $B(h)$ and $D(h)$ have weight $\theta$. The points $A(h), C(h)$, and $E(h)$ are $z$-aligned to the points $H_{i}(h), H_{j}(h)$, and $H_{k}(h)$, respectively. Point $A(h)$ is $y$-blocked by blocker $b_{A(h)}$ while point $E(h)$ is $x$-blocked by blocker $b_{E(h)}$. See Figure 1.

Exact location The exact location of the points comprising the clause gadget corresponding to clause $c(h)=\left(\neg V_{i} \vee \neg V_{j} \vee \neg V_{k}\right)$ and the corresponding connection points towards the variable gadget appears at Table 2 . 
Table 2: The points in the clause gadget associated with clause $c(h)=\left(\neg V_{i} \vee \neg V_{j} \vee \neg V_{k}\right)$ and the corresponding connection points.

\begin{tabular}{|c||c|c|c||c|}
\hline Point & $c_{x}$ & $c_{y}$ & $c_{z}$ & Weight \\
\hline \hline$A(h)$ & $1+i \epsilon$ & $1+h \epsilon+2 \epsilon / 3$ & $1-h \epsilon$ & 1 \\
$B(h)$ & $1+j \epsilon$ & $1+h \epsilon+2 \epsilon / 3$ & $1-h \epsilon$ & $\theta$ \\
$C(h)$ & $1+j \epsilon$ & $1+h \epsilon+\epsilon / 3$ & $1-h \epsilon$ & 1 \\
$D(h)$ & $1+k \epsilon$ & $1+h \epsilon+\epsilon / 3$ & $1-h \epsilon$ & $\theta$ \\
$E(h)$ & $1+k \epsilon$ & $1+h \epsilon$ & $1-h \epsilon$ & 1 \\
\hline$b_{A}(h)$ & $1+i \epsilon$ & $(1+\theta)^{2} / \theta$ & $1-h \epsilon$ & $\theta$ \\
$b_{E}(h)$ & $(1+\theta)^{2} / \theta$ & $1+h \epsilon$ & $1-h \epsilon$ & $\theta$ \\
\hline$H_{i}(h)$ & $1+i \epsilon$ & $1+h \epsilon+2 \epsilon / 3$ & 1 & $\theta$ \\
$H_{j}(h)$ & $1+j \epsilon$ & $1+h \epsilon+\epsilon / 3$ & 1 & $\theta$ \\
$H_{k}(h)$ & $1+k \epsilon$ & $1+h \epsilon$ & 1 & $\theta$ \\
\hline
\end{tabular}

Among the allocations in which the connection points $H_{i}(h), H_{j}(h)$ and $H_{k}(h)$ are allocated to players $y$ or $x$ (i.e., the clause gadget is non-breathing), the one that maximizes revenue leaves one of $A(h), C(h)$, and $E(h)$ unallocated. In contrast, if some of the connection points (say $H_{j}(h)$ ) is not allocated to players $x$ or $y$ (i.e., the clause gadget is breathing), the contribution of $A(h), C(h)$, and $E(h)$ can increase by allocating $A(h)$ and $B(h)$ to player $x$, $C(h)$ and $H_{j}(h)$ to $z$, and $D(h)$ and $E(h)$ to $y$. The other cases (i.e., when $H_{i}(h)$ or $H_{k}(h)$ are not allocated to the aforementioned players) have similar allocations of improved revenue.

An important property is that points in different clause gadgets are never aligned. This is achieved by dedicating a distinct $x z$-plane for the points in the gadget associated with any clause $c(h)=\left(V_{i} \vee V_{j} \vee V_{k}\right)$, and dedicating a distinct $x y$-plane for the points in the gadget associated with any clause $c(h)=\left(\neg V_{i} \vee \neg V_{j} \vee \neg V_{k}\right)$.

\section{The inapproximability result - The Analysis}

We show that the optimal revenue in the reduced instance $R(\Phi)$ of 3OptimalAuctionDeSIGN strongly depends on the maximum number of satisfied clauses in the original formula $\Phi$ of MAX-NM 3-SAT. Since approximating the second objective is hard, we show that approximating the first objective is also hard.

Consider a clause $c(h)$ consisting of variables $V_{i}, V_{j}$, and $V_{k}$, the corresponding gadget, and an allocation of variable points $v_{i}, v_{j}$, and $v_{k}$ to players $y$ and $z$. Our reduction relies on a relation between monotone allocations in $R(\Phi)$ and assignments for the variables in $\Phi$. In particular, we associate the allocation of a variable point to player $y$ (resp., to player $z$ ) with the assignment of value 1 (resp., 0 ) to its corresponding variable. Then, we can easily verify that the clause gadget associated with $c(h)$ is breathing if and only if the allocation of the variable points $v_{i}, v_{j}$, and $v_{k}$ implies an assignment that satisfies clause $c(h)$. Our bound follows by considering the gap in achievable revenue by a clause gadget depending on whether the corresponding clause is breathing or not.

Our analysis exploits a particular type of monotone allocations, defined as follows.

Definition 1 An allocation $A$ of instance $R(\Phi)$ is called simple if for every connection point that is allocated to a player, unallocating it violates monotonicity. 


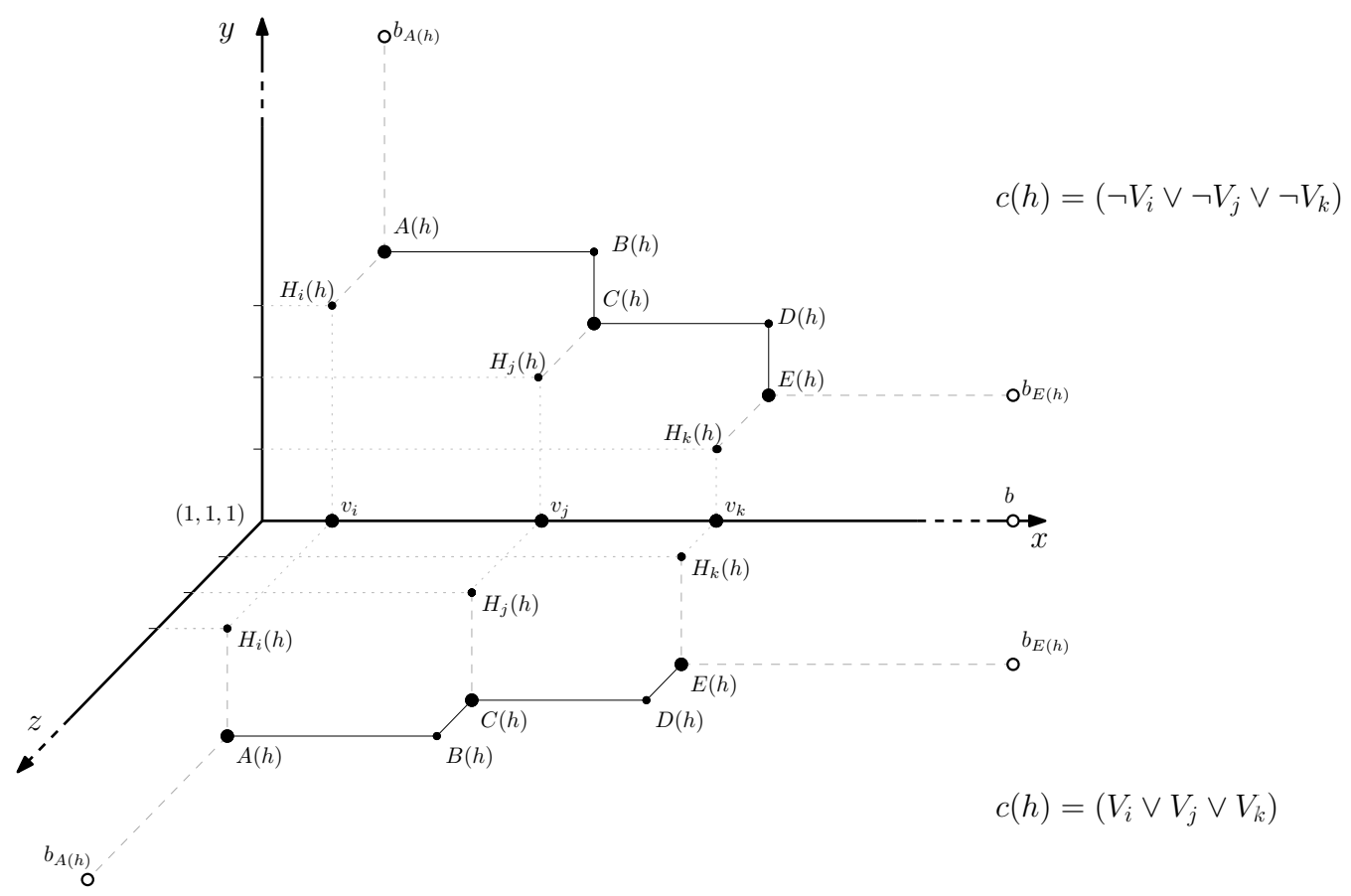

Figure 1: The clause gadgets corresponding to a clause $c(h)$ in the two cases where $c(h)=$ $\left(V_{i} \vee V_{j} \vee V_{k}\right)$ (set of points with $z$-coordinate higher than 1) and $c(h)=\left(\neg V_{i} \vee \neg V_{j} \vee \neg V_{k}\right)$ (set of points with $y$-coordinate higher than 1). Large black disks represent unit-weight or variable points, smaller black disks represent connection points or $\theta$-weight points, whereas white disks represent blockers.

Simple allocations are used as an intermediate step in the analysis and their correspondence to monotone allocations is revealed later. When accounting for the revenue of a simple allocation $A$, we use a simplified version that disregards the revenue obtained by connection points as well as non-blocker points with weight $\theta$; we refer to such points as $\theta$-weight points. We consider the revenue of the remaining points in several steps of our analysis and refer to it using the following definition.

Definition 2 The revenue of a simple allocation $A$ of $R(I)$ that is due (only) to variable points, blockers and unit-weight points in clause gadgets is called discounted revenue and is denoted by $\operatorname{drev}(A)$.

The following lemma bounds the discounted revenue derived by a clause gadget depending on whether it is breathing or not.

Lemma 1 Consider a simple allocation of maximum discounted revenue. If the gadget corresponding to a clause $c(h)$ is breathing, then its contribution to the discounted revenue is at least $5+3 \theta+2 \theta^{2}$ and at most $5+6 \theta+2 \theta^{2}$. If it is not breathing, its contribution to the discounted revenue is at least $4+4 \theta+2 \theta^{2}$ and at most $4+6 \theta+2 \theta^{2}$.

Proof. We account for the revenue from every clause gadget both in case it is breathing and in case it is non-breathing (see the description in Section 3). Note that the allocation of each 
gadget does not affect the allocation of any other gadget in the construction, since points in different clause gadgets are never aligned. Consider a clause $c(h)=\left(V_{i} \vee V_{j} \vee V_{k}\right)$ and the corresponding gadget (the case of $c(h)=\left(\neg V_{i} \vee \neg V_{j} \vee \neg V_{k}\right)$ is identical). It is not hard to see that the main part of the gadget (excluding the blockers) contributes at least $3-\theta$ and at most $3+2 \theta$ to the (maximum) discounted revenue if it is breathing, while it contributes at least 2 and at most $2+2 \theta$ if it is non-breathing. Also, each of the blockers in the clause gadgets contributes exactly $(1+\theta)^{2}$ to the revenue. We can now easily obtain each of the desired bounds by summing the corresponding terms. For example, the contribution of a breathing clause gadget to the (maximum) discounted revenue is at least $3-\theta+2(1+\theta)^{2}$ and it is at most $3+2 \theta+2(1+\theta)^{2}$, while the contribution of a non-breathing clause gadget to the (maximum) discounted revenue is at least $2+2(1+\theta)^{2}$ and it is at most $2+2 \theta+2(1+\theta)^{2}$.

Note that the definition of simple allocations implies that a connection point is allocated to a player only if the allocation of its aligned variable point or clause gadget point also enforces it to be allocated to the same player. In particular, if the variable point $v_{i}$ is not allocated, then the fact that the allocation is simple implies that all connection points aligned to $v_{i}$ are not enforced by $v_{i}$ and, hence, the clause gadgets corresponding to equations in which $V_{i}$ participates are all breathing. The next lemma implies that we only need to consider simple allocations where all variable points are allocated to some player (either $y$ or $z$ ).

Lemma 2 The simple allocation of maximum discounted revenue is complete, i.e., all variable points are allocated to players $y$ and $z$.

Proof. Consider a non-complete simple allocation in which variable point $v_{i}$ has not been allocated to any player. We allocate the variable point $v_{i}$ to the player among $y$ and $z$ that maximizes the number of breathing clause gadgets. Clearly, for every clause $c(h)=$ $\left(V_{i} \vee V_{j} \vee V_{k}\right)$ (similarly for $c(h)=\left(\neg V_{i} \vee \neg V_{j} \vee \neg V_{k}\right)$ ) such that some of the variable points $v_{j}$ and $v_{k}$ are not allocated, the gadget remains breathing no matter how the variable point $v_{i}$ is allocated. For every clause $c(h)$ such that variable points $v_{j}$ and $v_{k}$ are allocated (to players $y$ or $z$ ), there is an allocation of $v_{i}$ to either player $y$ or player $z$ that leaves the gadget corresponding to $c(h)$ breathing. Hence, there is an allocation of $v_{i}$ such that at most $d_{i} / 2$ clause gadgets become non-breathing. By Lemma 1, the contribution from these gadgets to the discounted revenue decreases by at most $(1+2 \theta) d_{i} / 2$. The contribution of the remaining (at most $d_{i}$ ) gadgets decreases by at most $3 \theta d_{i}$. Clearly, the total decrease in discounted revenue is smaller than the additional revenue $d_{i}(1 / 2+5 \theta)$ obtained by the allocation of $v_{i}$.

Now, we are ready to show the correspondence between the simple allocations that we have considered so far and the monotone allocations of instance $R(\Phi)$.

Lemma 3 For every monotone allocation $A$ with revenue $\operatorname{rev}(A)$, there is a complete simple allocation $A^{\prime}$ such that $\operatorname{drev}\left(A^{\prime}\right) \geq \operatorname{rev}(A)-10 m \theta$.

Proof. Given the monotone allocation $A$, we construct a simple allocation $A^{\prime \prime}$ by allocating variable points, clause gadget points of weight 1 , and blockers as in $A$. Among the connection points, the only ones that are allocated to some player (like in $A$ ) are the ones whose allocation is enforced. By Lemma 2, there is a complete simple allocation $A^{\prime}$ with discounted revenue $\operatorname{drev}\left(A^{\prime}\right) \geq \operatorname{drev}\left(A^{\prime \prime}\right)$. The lemma follows since the total number of connection points and 
$\theta$-weight points in clause gadgets is $5 \mathrm{~m}$ and the contribution of each of them to the revenue of $A$ cannot exceed $\theta(1+\theta)<2 \theta$.

Since $\theta$ has an extremely small value in our construction, it is clear that the optimal discounted revenue over complete simple allocations is a very good approximation of the optimal revenue over all monotone allocations. The proof of the next lemma exploits this observation.

Lemma 4 If the maximum number of satisfied clauses in $\Phi$ is $K$, then the revenue in the revenue-optimal monotone allocation of $R(\Phi)$ is between $(7-70 \theta) m+K$ and $(7+70 \theta) m+K$.

Proof. Let us consider an optimal assignment of values to the variables of $\Phi$ so that $K$ clauses are satisfied. We construct a complete simple allocation $A$ for $R(\Phi)$ as follows. For every variable $V_{i}$ that is set to 0 (resp., to 1 ), we allocate the variable point $v_{i}$ to player $z$ (resp., to player $y$ ). In this way, the variable points contribute $\sum_{i=1}^{n} d_{i}\left(\frac{1}{2}+5 \theta\right) \geq 3 \mathrm{~m} / 2$ to the discounted revenue of $A$. The blocker $b$ is allocated to player $x$ and contributes $\left(3 m\left(\frac{1}{2}+5 \theta\right)+\theta\right)(1+\theta) \geq 3 m / 2$ to the discounted revenue. Then, every clause gadget corresponding to a true (resp., false) clause is breathing (resp., non-breathing). The points in the clause gadgets are allocated so that their contribution to the discounted revenue is as high as possible. By Lemma 1, we have that the contribution of a breathing clause gadget to the discounted revenue is at least $5+3 \theta+2 \theta^{2} \geq 5$. For each clause that is false, the corresponding clause gadget is non-breathing. Hence, its contribution to the discounted revenue is at least $4+4 \theta+2 \theta^{2} \geq 4$. So, the total discounted revenue is at least

$$
3 m / 2+3 m / 2+5 K+4(m-K) \geq(7-70 \theta) m+K .
$$

Clearly, the right-hand side of this inequality is a lower bound on the revenue of the revenueoptimal monotone allocation as well.

Now, consider a complete simple allocation of maximum discounted revenue and the assignment of values to the variables $V_{i}$ this allocation implies. Consider the clauses whose corresponding gadgets are breathing. By our construction, this implies that the clauses are satisfied by the assignment; so, there are at most $K$ such clauses and the remaining $m-K$ clause gadgets are non-breathing. In total, using Lemma 1, the discounted revenue of the complete simple allocation is at most

$$
\begin{aligned}
3 m\left(\frac{1}{2}+5 \theta\right)+\left(3 m\left(\frac{1}{2}+5 \theta\right)+\theta\right) & (1+\theta)+K\left(5+6 \theta+2 \theta^{2}\right)+(m-K)\left(4+6 \theta+2 \theta^{2}\right) \\
& <(7+60 \theta) m+K .
\end{aligned}
$$

Hence, by Lemma 3 , the revenue-optimal monotone allocation has revenue at most $(7+$ $70 \theta) m+K$.

We are ready to prove our main result.

Theorem 1 For every constant $\delta \in(0,1 / 2)$, it is NP-hard to approximate 3OptimalAuCTIONDESIGN within a factor $\frac{63+\delta}{64-\delta}$.

Proof. Let $\delta \in(0,1 / 2), \eta=\delta / 10$ and $\theta=\delta / 2800$. We know from [Guruswami and Khot, $2005]$ that given $\Phi$, it is NP-hard to distinguish cases where the maximum number of satisfied 
clauses is at least $(1-\eta) m$ or at most $(7 / 8+\eta) m$, Lemma 4 implies that distinguishing between cases where the maximum revenue among all monotone allocations of $R(\Phi)$ is at least $(7-70 \theta) m+(1-\eta) m=(64-\delta) m / 8$ and at most $(7+70 \theta) m+(7 / 8+\eta) m=(63+\delta) m / 8$ is NP-hard as well.

\section{Deterministic vs randomized auctions}

We now present the upper bound on the revenue-gap between deterministic and randomized mechanisms.

Theorem 2 The revenue obtained by the optimal deterministic truthful mechanism can be at most $\frac{13}{14}$ of the revenue obtained by the optimal truthful-in-expectation mechanism.

Proof. We present an instance of 3OptimalAuctionDesign such that the revenue-maximizing deterministic monotone allocation yields revenue at most 13/14 of the revenue that can be derived by a randomized monotone allocation. The main part of our construction consists of 15 points, the coordinates of which are in $(1-\theta, 1+\theta)$, for some arbitrarily small $\theta>0$. Additionally, there are 7 points which we call blockers and have identical properties to the blockers described in Section 3.1. Each blocker is $x-, y$-, or $z$-aligned to some other point and its corresponding coordinate is exceptionally large. The weights and coordinates of all points are such that blockers are always allocated towards their "preferred" direction and points that are blocked are never allocated towards their blocked direction, at any deterministic revenue-optimal allocation.

Again, we use the notation $p_{(+x)} q$ and $p_{(-x)} q$, for two $x$-aligned points $p$ and $q$ of the main part of the construction, to denote that $q$ has larger and smaller $x$-coordinate than $p$, respectively (similarly for the other coordinates). The main part of our construction consists of two separate 7 -sequences of points $P$ and $Q$, and an extra point $u$ that connects them. We begin with a description of the relative position of the points of our construction and then give the exact coordinates in Table 3 (see also Figure 2).

Gadget $P$ Let $P$ : $\left[p_{1(-y)} p_{2(+z)} p_{3(-x)} p_{4(+y)} p_{5(-z)} p_{6(+y)} p_{7}\right]$ be such that $p_{1}$ and $p_{7}$ are $x$ aligned. Points $p_{2}, p_{4}$, and $p_{6}$ have weight 1 , while points $p_{1}, p_{3}, p_{5}$, and $p_{7}$ have weight $\theta$. Point $p_{2}$ is $x$-blocked by blocker $b\left(p_{2}\right)$, point $p_{4}$ is $z$-blocked by blocker $b\left(p_{4}\right)$, and point $p_{6}$ is $x$-blocked by blocker $b\left(p_{6}\right)$. Among the deterministic monotone allocations in which points $p_{1}$ and $p_{7}$ are allocated to player $x$ or $z$, the one that maximizes revenue should leave at least one of the points $p_{2}, p_{4}$, and $p_{6}$ unallocated. In contrast, if for example $p_{1}$ is not allocated to either $x$ or $z$, the contribution of $p_{2}, p_{4}$, and $p_{6}$ can increase by allocating $p_{2}$ to player $y, p_{4}$ to player $x$, and $p_{6}$ to player $z$. The case where $p_{7}$ is allocated to neither $x$ nor $z$ admits a similar allocation of improved revenue.

Gadget $Q$ Let $Q:\left[q_{1(-z)} q_{2(+x)} q_{3(-y)} q_{4(+z)} q_{5(-x)} q_{6(+z)} q_{7}\right]$ be such that $q_{1}$ and $q_{7}$ are $y$ aligned. Points $q_{2}, q_{4}$, and $q_{6}$ have weight 1 , while points $q_{1}, q_{3}, q_{5}$, and $q_{7}$ have weight $\theta$. Point $q_{2}$ is $y$-blocked by blocker $b\left(q_{2}\right)$, point $q_{4}$ is $x$-blocked by blocker $b\left(q_{4}\right)$, and point $q_{6}$ is $y$-blocked by blocker $b\left(q_{6}\right)$. Among the deterministic monotone allocations in which both points $q_{1}$ and $q_{7}$ are allocated to player $y$ or $x$, the one that maximizes revenue should leave at least one of the points $q_{2}, q_{4}$, and $q_{6}$ unallocated. In contrast, 
if for example $q_{1}$ is not allocated to either $y$ or $x$, the contribution of $q_{2}, q_{4}$, and $q_{6}$ can increase by allocating $q_{2}$ to player $z, q_{4}$ to player $y$, and $q_{6}$ to player $x$. The case where $q_{7}$ is allocated to neither $y$ nor $x$ admits a similar allocation of improved revenue.

Point $u$ Let $u$ be a point with weight 1 such that $u$ is $y$-aligned with $q_{1}$ and $q_{7}$ and has a smaller $y$-coordinate than each of them, and, moreover, $u$ is $x$-aligned with $p_{1}$ and $p_{7}$ and has a smaller $x$-coordinate than each of them. Point $u$ is $z$-blocked by blocker $b(u)$.

Table 3: The construction in the proof of Theorem 2.

\begin{tabular}{|c||c|c|c||c|}
\hline Point & $c_{x}$ & $c_{y}$ & $c_{z}$ & wgt \\
\hline \hline$p_{1}$ & $1+3 \theta / 4$ & 1 & 1 & $\theta$ \\
\hline$p_{2}$ & $1+3 \theta / 4$ & $1-2 \theta / 3$ & 1 & 1 \\
\hline$b\left(p_{2}\right)$ & $(1+\theta)^{2} / \theta$ & $1-2 \theta / 3$ & 1 & $\theta$ \\
\hline$p_{3}$ & $1+3 \theta / 4$ & $1-2 \theta / 3$ & $1+\theta / 3$ & $\theta$ \\
\hline$p_{4}$ & $1+\theta / 2$ & $1-2 \theta / 3$ & $1+\theta / 3$ & 1 \\
\hline$b\left(p_{4}\right)$ & $1+\theta / 2$ & $1-2 \theta / 3$ & $(1+\theta)^{2} / \theta$ & $\theta$ \\
\hline$p_{5}$ & $1+\theta / 2$ & $1-\theta / 3$ & $1+\theta / 3$ & $\theta$ \\
\hline$p_{6}$ & $1+\theta / 2$ & $1-\theta / 3$ & 1 & 1 \\
\hline$b\left(p_{6}\right)$ & $(1+\theta)^{2} / \theta$ & $1-\theta / 3$ & 1 & $\theta$ \\
\hline$p_{7}$ & $1+\theta / 2$ & 1 & 1 & $\theta$ \\
\hline$q_{1}$ & 1 & $1+2 \theta / 3$ & 1 & $\theta$ \\
\hline$q_{2}$ & 1 & $1+2 \theta / 3$ & $1-2 \theta / 3$ & 1 \\
\hline$b\left(q_{2}\right)$ & 1 & $(1+\theta)^{2} / \theta$ & $1-2 \theta / 3$ & $\theta$ \\
\hline$q_{3}$ & $1+\theta / 4$ & $1+2 \theta / 3$ & $1-2 \theta / 3$ & $\theta$ \\
\hline$q_{4}$ & $1+\theta / 4$ & $1+\theta / 3$ & $1-2 \theta / 3$ & 1 \\
\hline$b\left(q_{4}\right)$ & $(1+\theta)^{2} / \theta$ & $1+\theta / 3$ & $1-2 \theta / 3$ & $\theta$ \\
\hline$q_{5}$ & $1+\theta / 4$ & $1+\theta / 3$ & $1-\theta / 3$ & $\theta$ \\
\hline$q_{6}$ & 1 & $1+\theta / 3$ & $1-\theta / 3$ & 1 \\
\hline$b\left(q_{6}\right)$ & 1 & $(1+\theta)^{2} / \theta$ & $1-\theta / 3$ & $\theta$ \\
\hline$q_{7}$ & 1 & $1+\theta / 3$ & 1 & $\theta$ \\
\hline$u$ & 1 & 1 & 1 & 1 \\
\hline$b(u)$ & 1 & 1 & $(1+\theta)^{2} / \theta$ & $\theta$ \\
\hline & & & & \\
\hline
\end{tabular}

Let us examine the possible deterministic allocations of the above construction. If $u$ is not allocated the maximum revenue does not exceed $7(1+\theta)^{2}+(6+8 \theta)(1+\theta) \leq 13+28 \theta+15 \theta^{2}$. Otherwise, if $u$ is allocated to player $y$ or $x$, some of the other unit-weight points can not be allocated to a non-blocked direction/player. To see why this is true, assume that $u$ is allocated to player $y$ (the case that $u$ is allocated to player $x$ is symmetric). Then, points $q_{2}$ and $q_{6}$ can only be allocated to player $x$ in a monotone allocation, thus point $q_{4}$ can not be allocated to any of its non-blocked directions. Again, the maximum revenue does not exceed $13+28 \theta+15 \theta^{2}$.

Now, consider the randomized allocation in which the blockers are allocated to their preferred direction, the unit-weight points are allocated equiprobably between their two nonblocked directions, and the remaining points are allocated as follows: points $q_{1}, q_{7}$, and $p_{5}$ are allocated equiprobably between players $y$ and $z$, points $p_{1}, p_{7}$, and $q_{3}$ are allocated equiprobably between players $x$ and $y$, and points $q_{5}$ and $p_{3}$ are allocated equiprobably between players 


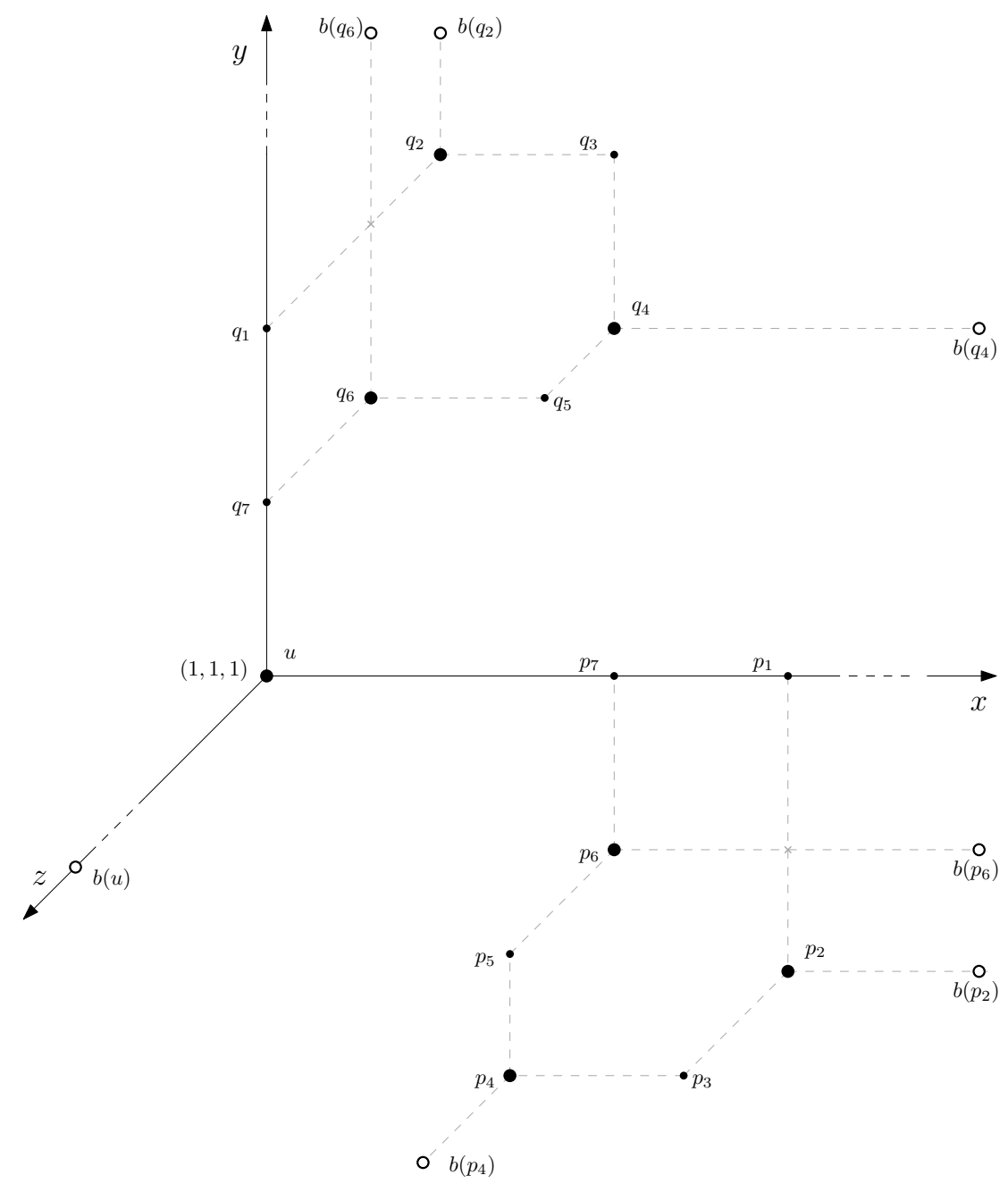

Figure 2: The instance used in the proof of Theorem 2. Large black disks represent the unit-weight points and smaller black disks represent points of weight $\theta$ that belong to the main part of the construction. White disks denote blockers. 
$x$ and $z$. Regarding the payments, it suffices to consider that the payment of some player, e.g., $x$, that is allocated a fraction $f_{x}$ of some point $p$ is at least the lowest $x$-coordinate among the $x$-aligned points to $p$ that have non-zero probability to be allocated to $x$ times $f_{x}$ (see the discussion after Observation 1). It can be easily verified that this is a monotone allocation with revenue at least $7(1+\theta)^{2}+(7+8 \theta)(1-\theta) \geq 14$. The theorem follows since $\theta$ can take any arbitrarily small positive value.

\section{References}

Caragiannis, I., Kaklamanis, C., and Kyropoulou, M. (2013). Limitations of deterministic auction design for correlated bidders. In Proceedings of the 21st Annual European Symposium on Algorithms, ESA '13, pages 277-288.

Chen, X., Hu, G., Lu, P., and Wang, L. (2011). On the approximation ratio of $k$-lookahead auction. In Proceedings of the 7th International Conference on Internet and Network Economics, WINE '11, pages 61-71.

Constantin, F., Ito, T., and Parkes, D. C. (2007). Online auctions for bidders with interdependent values. In Proceedings of the 6th International Joint Conference on Autonomous Agents and Multiagent Systems, AAMAS '07, pages 110:1-110:3.

Crémer, J. and McLean, R. P. (1985). Optimal selling strategies under uncertainty for a discriminating monopolist when demands are interdependent. Econometrica, 53(2):345361.

Crémer, J. and McLean, R. P. (1988). Full extraction of the surplus in bayesian and dominant strategy auctions. Econometrica, 56(6):1247-1257.

Diakonikolas, I., Papadimitriou, C., Pierrakos, G., and Singer, Y. (2012). Efficiency-revenue trade-offs in auctions. In Proceedings of the 39th International Colloquium Conference on Automata, Languages, and Programming - Volume Part II, ICALP '12, pages 488-499.

Dobzinski, S., Fu, H., and Kleinberg, R. D. (2011). Optimal auctions with correlated bidders are easy. In Proceedings of the 43rd Annual ACM Symposium on Theory of Computing, STOC '11, pages 129-138.

Esö, P. (2005). An optimal auction with correlated values and risk aversion. J. Economic Theory, 125(1):78-89.

Guruswami, V. and Khot, S. (2005). Hardness of max 3sat with no mixed clauses. In Proceedings of the 20th Annual IEEE Conference on Computational Complexity, CCC '05, pages $154-162$.

Krishna, V. (2009). Auction Theory. Academic Press.

Levin, D. and Smith, J. L. (1996). Optimal reservation prices in auctions. The Economic Journal, 106(438):1271-1283.

Maskin, E. (2003). Auctions and efficiency. In Dewatripont, M., Hansen, L. P., and Turnovsky, S. J., editors, Advances in Economics and Econometrics: Theory and Applications, Eighth World Congress (Econometric Society Monographs), volume 3, pages 1-24. 
Milgrom, P. R. and Weber, R. J. (1982). A theory of auctions and competitive bidding. Econometrica, 50(5):1089-1122.

Myerson, R. B. (1981). Optimal auction design. Mathematics of Operations Research, 6(1):5873.

Nisan, N., Roughgarden, T., Tardos, É., and Vazirani, V. V. (2007). Algorithmic Game Theory. Cambridge University Press, New York, NY.

Papadimitriou, C. H. and Pierrakos, G. (2011). On optimal single-item auctions. In Proceedings of the 43rd Annual ACM Symposium on Theory of Computing, STOC '11, pages $119-128$.

Ronen, A. (2001). On approximating optimal auctions. In Proceedings of the 3rd ACM Conference on Electronic Commerce, EC '01, pages 11-17.

Ronen, A. and Saberi, A. (2002). On the hardness of optimal auctions. In Proceedings of the 43rd Symposium on Foundations of Computer Science, FOCS '02, pages 396-405.

Roughgarden, T. and Talgam-Cohen, I. (2013). Optimal and near-optimal mechanism design with interdependent values. In Proceedings of the 14th ACM Conference on Electronic Commerce, EC '13, pages 767-784. 


\section{A Proof of Observation 1}

Let $\mathcal{M}=(x, \mathbf{p})$ be a truthful in expectation mechanism. We will first prove that allocation $x$ is monotone. Truthfulness in expectation implies that the following two inequalities hold for any $i, \mathbf{v}_{-i}, v_{i}$ and $v_{i}^{\prime}$ : (To simplify notation, we remove index $i$ and the fixed argument $\mathbf{v}_{-i}$.)

$$
x(v) \cdot v-p(v) \geq x\left(v^{\prime}\right) \cdot v-p\left(v^{\prime}\right),
$$

and

$$
x\left(v^{\prime}\right) \cdot v^{\prime}-p\left(v^{\prime}\right) \geq x(v) \cdot v^{\prime}-p(v) .
$$

By combining inequalities (2) and (3) we get

$$
x(v) \cdot v+x\left(v^{\prime}\right) \cdot v^{\prime} \geq x\left(v^{\prime}\right) \cdot v+x(v) \cdot v^{\prime} .
$$

The above inequality implies that

$$
\left(x(v)-x\left(v^{\prime}\right)\right)\left(v-v^{\prime}\right) \geq 0,
$$

which, in turn, implies monotonicity.

Now, consider some fixed monotone allocation $x$. We will prove that assigning payments

$$
p\left(v_{\lambda}\right)=x\left(v_{\lambda}\right) \cdot v_{\lambda}-\sum_{k=1}^{\lambda-1} x\left(v_{k}\right) \cdot\left(v_{k+1}-v_{k}\right),
$$

yields the maximum achievable revenue by a truthful in expectation mechanism $\mathcal{M}$, where $v_{\lambda}$ denotes the $\lambda$-th possible valuation of $i$ such that $\left(v_{\lambda}, \mathbf{v}_{-i}\right)$ occurs with positive probability, for fixed $i$ and $\mathbf{v}_{-i}$. Inequality (2) implies the following upper bound on the payments of $\mathcal{M}$, i.e, for any fixed $i$, and $\mathbf{v}_{-i}$ and for any $v$ and $v^{\prime}$, it holds that

$$
p(v) \leq x(v) \cdot v-x\left(v^{\prime}\right) \cdot v+p\left(v^{\prime}\right) .
$$

Note that for fixed $i, \mathbf{v}_{-i}$, we can compute a tight upper bound on $p\left(v_{\lambda}\right)$ for every $\lambda$ by replacing inequality (5) with an equality and considering it as a recursion on $\lambda$ with $p\left(v_{0}\right)=0$, i.e., $p\left(v_{\lambda}\right)=x\left(v_{\lambda}\right) \cdot v_{\lambda}-x\left(v_{\lambda-1}\right) \cdot v_{\lambda}+p\left(v_{\lambda-1}\right)$. In this way, we obtain the payments defined by equality (4), which we denote by p. Now, assume that there exists a set of (different) payments $\mathbf{p}^{\prime}$, such that $\mathcal{M}^{\prime}=\left(x, \mathbf{p}^{\prime}\right)$ is truthful in expectation and achieves higher revenue than $\mathcal{M}=(x, \mathbf{p})$. It holds that there exists a valuation vector $\left(v_{w}, \mathbf{v}_{-i}\right)$ such that (for some player $i) p^{\prime}\left(v_{w}\right)>p\left(v_{w}\right)$. Consider the smallest such valuation $v_{m}$, i.e., $p^{\prime}\left(v_{m}\right)>p\left(v_{m}\right)$ and $p^{\prime}\left(v_{m-1}\right) \leq p\left(v_{m-1}\right)$. It holds that

$$
\begin{aligned}
p\left(v_{m}\right) & <p^{\prime}\left(v_{m}\right) \\
& \leq x\left(v_{m}\right) \cdot v_{m}-x\left(v_{m-1}\right) \cdot v_{m}+p^{\prime}\left(v_{m-1}\right) \\
& \leq x\left(v_{m}\right) \cdot v_{m}-x\left(v_{m-1}\right) \cdot v_{m}+p\left(v_{m-1}\right) \\
& =x\left(v_{m}\right) \cdot v_{m}-\sum_{k=1}^{m-1} x\left(v_{k}\right) \cdot\left(v_{k+1}-v_{k}\right),
\end{aligned}
$$

where the second inequality follows by inequality (5), since $\mathcal{M}^{\prime}$ is truthful in expectation, and the equality follows by substituting $p\left(v_{m-1}\right)$ using (4). However, the above strict inequality 
regarding $p\left(v_{m}\right)$ contradicts the fact that $\mathbf{p}$ is defined by equality (4). We conclude that the payments defined by equality (4) yield the maximum revenue that can be achieved by a truthful in expectation mechanism that applies a given allocation $x$.

To prove our final claim, we consider a mechanism $\mathcal{M}=(x, \mathbf{p})$ such that allocation $x$ is monotone and $\mathbf{p}$ is defined by equality (4). We will prove that $\mathcal{M}=(x, \mathbf{p})$ is truthful in expectation. It suffices to prove inequality (2), which is equivalent to showing that for any $i$, $\mathbf{v}_{-i}, v_{\lambda}$ and $v_{\lambda^{\prime}}$ it holds that

$$
x\left(v_{\lambda}\right) \cdot v_{\lambda}-p\left(v_{\lambda}\right) \geq x\left(v_{\lambda^{\prime}}\right) \cdot v_{\lambda}-p\left(v_{\lambda^{\prime}}\right) .
$$

By substituting the payments using equality (4), we get

$$
\sum_{k=1}^{\lambda-1} x\left(v_{k}\right) \cdot\left(v_{k+1}-v_{k}\right) \geq x\left(v_{\lambda^{\prime}}\right) \cdot v_{\lambda}-x\left(v_{\lambda^{\prime}}\right) \cdot v_{\lambda^{\prime}}+\sum_{k=1}^{\lambda^{\prime}-1} x\left(v_{k}\right) \cdot\left(v_{k+1}-v_{k}\right) .
$$

We distinguish between two cases depending on the relative order of $\lambda$ and $\lambda^{\prime}$. If $\lambda \geq \lambda^{\prime}$ it holds that

$$
\begin{aligned}
\sum_{k=\lambda^{\prime}}^{\lambda-1} x\left(v_{k}\right) \cdot\left(v_{k+1}-v_{k}\right) & \geq \sum_{k=\lambda^{\prime}}^{\lambda-1} x\left(v_{\lambda^{\prime}}\right) \cdot\left(v_{k+1}-v_{k}\right) \\
& =x\left(v_{\lambda^{\prime}}\right) \cdot\left(v_{\lambda}-v_{\lambda^{\prime}}\right)
\end{aligned}
$$

where inequality (6) holds by monotonicity and the fact that $\lambda \geq \lambda^{\prime}$ implies $v_{\lambda} \geq v_{\lambda^{\prime}}$. Otherwise, if $\lambda<\lambda^{\prime}$ it holds that

$$
\begin{aligned}
\sum_{k=\lambda}^{\lambda^{\prime}-1} x\left(v_{k}\right) \cdot\left(v_{k+1}-v_{k}\right) & \leq \sum_{k=\lambda}^{\lambda^{\prime}-1} x\left(v_{\lambda^{\prime}}\right) \cdot\left(v_{k+1}-v_{k}\right) \\
& =x\left(v_{\lambda^{\prime}}\right) \cdot\left(v_{\lambda^{\prime}}-v_{\lambda}\right),
\end{aligned}
$$

and the proof is complete. 